\title{
A Review Article of Satellite Image Different Type Approach
}

\author{
Kritika Dshmukh ${ }^{1}$, Meena Badole ${ }^{2}$ \\ ${ }^{1}$ M. Tech Student Department of Electronic \& Communication, LNCT Indore (M.P.), India \\ ${ }^{2}$ Assistant Professor, Department of Electronic \& Communication, LNCT Indore (M.P.), India
}

\begin{abstract}
This paper a survey of the automatic detection of the region of interest (ROI) in Histopathologoical images by means of advanced segmentation techniques. The clustering techniques like fuzzy C-means (FCM) and K-means algorithms for the color image segmentation are implemented on a dataset which consisted of skin images. Euclidian distance is used as a distance metric in both the algorithms. The resultant segmented regions which were obtained from these two techniques were compared and found to be of similar features. The entire experiment is implemented in the $L^{*} a^{*} b^{*}$ color space and a concise discussion regarding the color space conversion is carried out. This paper presents a detailed discussion on the various steps of the two clustering techniques and its requirements and shows how the result obtained from them could be used for diagnosis purpose by the human experts in medical treatment. Due to the increasing requirements for transmission of images in computer, mobile environments, the research in the field of image compression has increased significantly. Image compression plays a crucial role in digital image processing, it is also very important for efficient transmission and storage of images
\end{abstract}

Keywords: Region of interest, K-means, Fuzzy C-Means, L*a*b* color space

\section{Introduction}

The first step for Region of Interest (ROI) segmentation of the digital images is usually the Pattern Recognition (PR) procedure. Segmentation process basically deals with the step of fragmenting or segmenting the entire image into no overlapping and uniform regions to discover certain meaningful information from the segmented regions which are generally considered to be homogenous based on various criteria like motion, color, texture etc. which makes the segmentation the leading step or procedure in the researches that leads to image processing. In addition to it an unsupervised study as clustering is also carried out where the various data items are grouped together after partitioning keeping in mind the following 2 properties: (1) High Cohesion (2) Low coupling. The first property stands for the fact that the whichever data item belong to one particular cluster has to possess high similarities whereas low coupling stands for the fact that two different clusters should have two different class of data items.

Image segmentation is that the drawback of partitioning a picture into significant regions on the premise of grey-level, color, texture. this means the generality of the problemsegmentation may be found in any image-driven method, e.g. fingerprint/text/face recognition, trailing of moving people/cars/airplanes, etc. for several applications, segmentation reduces to finding Associate in Nursing object in a picture. This involves partitioning the image into 2 categories of regions - either object or background. it's merely not possible in apply to manually method all the pictures (like magnetic resonance imaging and CT scan), owing to the overwhelming quantity of data it provides. Therefore we have a tendency to style algorithms that search for bound patterns and objects of interest and place them to our attention.
One of many techniques under image processing is image compression. Image compression has many applications and plays an important role in efficient transmission and storage of images. The image compression aims at reducing redundancy in image data to store or transmit only a minimal number of samples And from this we can reconstruct a good accession of the original image in accordance with human visual perception

\subsection{Literature Survey and Issues of Old Articles}

The potential of intelligent knowledge analysis techniques has up with the increasing quantity of knowledge on the market digitally. With enhancements in laptop performance and development of the digital devices opportunities are created to use multimedia system knowledge, admire pictures and voice. In existing storage systems, a amount of \{information that our system is ready to store associated an index entry is created once information is keep. Once users wish to retrieve some item of data, they use the index to seek out the specified item. it's tough to seek out one thing accurately and quickly from among the various complicated things in an exceedingly information due to the massive index house for the info being searched.[3]

1)Computerization of the entire system forms a major part of the ongoing researches in medical and technology in the domain of ROI extraction. Such an approach involves down sampling of the input[4] in which a lower resolution input is utilized for the extraction of the various features that are based on color coding and sparse coding of the sub patches and then they are then categorized and classified to detect the ROI using Support Vector machines(SVM).

2)In a similar attempt, HOG $3 D$ descriptor for initializing the agents are used on images by Yassine et. al [12] which exhibits a complete automated approach for extraction of ROI which is dependent on multivalent system 


\section{International Journal of Science and Research (IJSR) \\ ISSN (Online): 2319-7064}

Index Copernicus Value (2015): 78.96 | Impact Factor (2015): 6.391

incorporating spratio temporal interest point detection in the renal region. It also discussed about a 'Semi-automatic approach' this consists of various methods that enables drawing of the renal ROI considering an initial point.

\subsection{Fundamental of Segmentation Technique}

Segmentation technique may be divided roughly into the subsequent categories:

1) Thresholding approaches, (6) Artificial neural networks,

2) Region growing approaches, (7) Deformable models, and

3) Classifiers, (8) Atlas guided approaches.

4) Bunch2 approaches,

5) Andrei Markov random field models,

Different notable ways conjointly exist of the various approaches expressed above; thresholding, classifier, clustering, and Andrei Markov random field approaches may be thought of component classification ways.[2]

\subsection{Basic of Algorithms}

Three normally used agglomeration algorithms area unit the 1) k-means,

2) The fuzzy c-means algorithmic rule,

3) The expectation-maximization (EM) algorithmic rule.

Within the k-means agglomeration algorithmic rule clusters mean is iteratively computed and a mean intensity every for every\} category is assigned and image is mesmeric the by assignment each component within the category with the nearest mean. The fuzzy c-means algorithmic rule generalizes the $\mathrm{k}$-means algorithmic rule, giving soft segmentations supported fuzzy pure mathematics. Coaching knowledge isn't needed by agglomeration algorithms, however they are doing need associate initial segmentation (or equivalently, initial parameters). Therefore, not like classifier ways, agglomeration algorithms may be sensitive to noise and intensity in homogeneities. This lack of special modeling, however, will give important benefits for quick computation.[2]

\subsection{Disadvantages of FCM and K-Means}

These methods use the minimum distance clustering algorithm as a clustering system. In these methods, input data is treated as multi-dimensional vectors, the degree of similarity between input data is expressed as a distance (e.g., the Euclidean distance), and the classification of the input data is done using these distances.

1) FCM is higherlly complex and higher noise

2) K-mean clustering approach lower complexity and higher efficient statistical data to provide.

3) These are very iterative method

4) K -mean approach easily implemented

5) $\mathrm{K}$ value input to algorithm these are dependent for user selecting

6) Work only numerical data on any image

7) Gives hierarchal clustering.

\subsection{Basics of Different Type of Algorithem}

1. Marker-Controlled Watershed Segmentation Method

This example shows how to use watershed segmentation to separate touching objects in an image. The watershed transform is often applied to this problem. Properties of water Segmentation method

1) These are based on the principle of morphology

2) And high capture region.

3) Intensity the intensity of any object

4) Using the gray color contrast tumor and back ground of image

5) Watershed method is a suitable approach to find out the tumor

6) These are tracking the light and dark pixel to converting for high and low intensity for a lightness These are remove noise and highly efficient method.

\section{Texture Segmentation Using Texture Filters using}

This example shows how to use texture segmentation to identify regions based on their texture. Your goal is to segment two kinds of fabric in an image using texture filters. Properties of Texture Segmentation method

1) Accuracy to increasing by using high pass filter method

2) Edge detection are also below pass filter applied to detecting of brain tumor

3) This method increasing the boundaries intensity of any object

4) Sharpening to improve images.

\section{Enhance result in Based Segmentation Using K-Means Clustering Method}

This example shows how to segment colors in an automated fashion using the $\mathrm{L}^{*} \mathrm{a} \mathrm{b}^{*}$ color space and K-means

clustering.

1) In this method to create suitable gape of higher and lower intensity of any tumor cell and sub cell.

2) These are removed the white Gaussian noise of any image

3) These methods are also called by the opening by reconstruction and closing by reconstruction.

4) Statically to removed the noise.

\section{Compression Technique}

\section{1) (DCT) Discrete Cosine Transform Image compression}

The most popular technique for image compression, over the past several years, was discrete cosine transform (DCT). Its selection as the standard for JPEG is One of the major reasons for its popularity. DCT is used by many Nonanalytical applications such as image processing and signalprocessing DSP applications such as video conferencing. The DCT is used in transformation for data compression. DCT is an orthogonal transform, which has a fixed set of basis function.Dct is used to map an image space into a frequency.[15] DCT has many

\section{Advantages:}

1)It has the ability to pack energy in the lower frequencies for image data.

2)It has the ability to reduce the blocking artefact effect and this effect results from the boundaries between sub-images 


\section{International Journal of Science and Research (IJSR) \\ ISSN (Online): 2319-7064}

Index Copernicus Value (2015): 78.96 | Impact Factor (2015): 6.391

become visible

\section{2) Artificial Neural Networks}

Learning ability: an ANN learns by example; it extracts information from the training data without need for rules or formulae resulting in less need to determine relevant factors a priori. The ANN can adapt more easily to new conditions, i.e. input data, than conventional algorithms. Distributed knowledge: an ANN stores knowledge distributive in the weights of its neurons. This architecture suits parallel processing. Parallelism: an ANN consists of a large number of interconnected simple processing units, i.e. neurons, operating in parallel. This structure is very suitable for parallel processing, for example on transporter systems. However, the design must limit the amount of communication in order to lead to a practical system

\section{3) HAAR Wavelet Image Compression}

The objective of our project was to perform the discrete Haar wavelet transformation on an image for the purpose of compression. The Haar wavelet transformation is composed of a sequence of low-pass and high-pass filters, known as a filter bank. These filter sequences can be applied in the same way as a discrete FIR filter in the DSP, using the MACP command, except as multiple successive FIR filters. The low pass filter performs an averaging/blurring operation, and is expressed as:

$$
H=\frac{1}{\sqrt{2}}(1,1)
$$

and the high-pass filter performs a differencing operation and can be expressed as:

$$
G=\frac{1}{\sqrt{2}}(1,-1)
$$

on any adjacent pixel pair. The complete wavelet transform can be represented in matrix format by:

\section{Algorithm Flow Diagram}

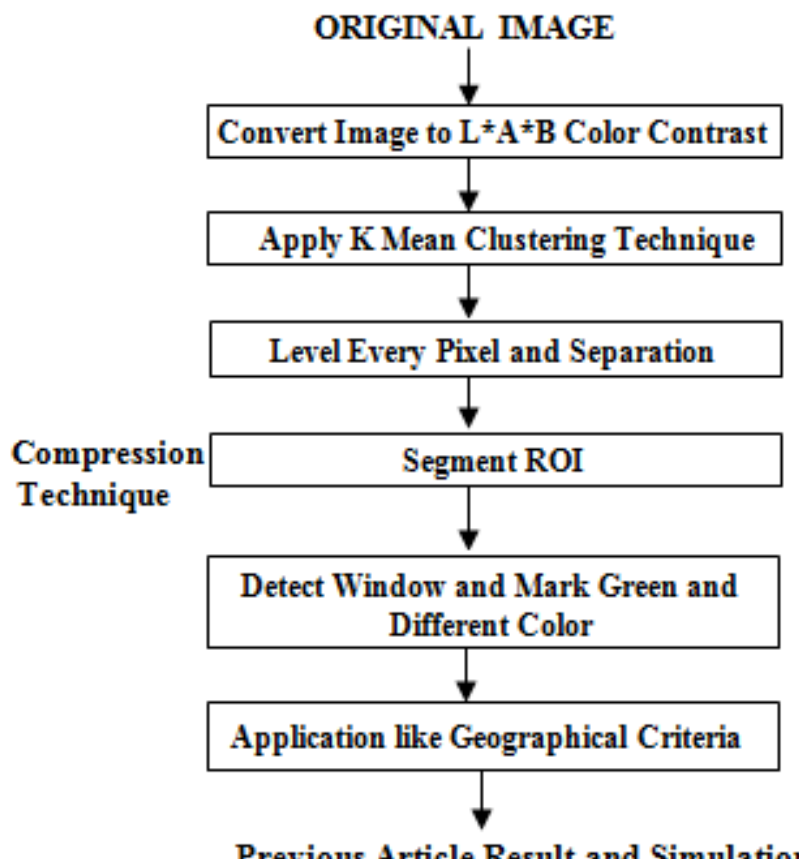

Previous Article Result and Simulation
1) In terms of image accuracy

Later the images would go through a Modified Histogram Clustering - Color Threshold technique to identify the position of the tumor. According to various Tempered image scan images, the threshold values of the image's HSV for the tumor happens to be common and lies between these values:

$\begin{array}{lll}\text { HSV } & \text { High (\%) } & \text { Low (\%) } \\ \text { Hue } & 23 & 10 \\ \text { Saturation } & 70 & 07 \\ \text { Value } & 100 & 70\end{array}$

We used 50 neuro images to optimize our system and 100 out-of-sample neuro images to test our system. The proposed tumor detection and localization system was found to be able to accurately detect and localize Tempered image in magnetic resonance imaging. The proposed system achieved an error rate of $8 \%$ in identifying and localizing tumors.

\section{2) In terms of noise reduction of images}

The proposed brain tumor detection and localization framework comprises five steps: image acquisition, preprocessing, edge detection, modified histogram clustering and morphological operations. After morphological operations, tumors appear as pure white color on pure black backgrounds. We used 50 neuro images to optimize our system and 100 out-of-sample neuro images to test our system.

\section{3) In terms of efficiency}

The proposed tumor detection and localization system was found to be able to accurately detect and localize brain tumor in magnetic resonance imaging. This system achieved an error rate of $8 \%$. The preliminary results demonstrate how a simple machine learning classifier with a set of simple image-based features can result in high classification accuracy. The preliminary results also demonstrate the efficacy and efficiency of our five-step brain tumor detection and localization approach and motivate us to extend this framework to detect and localize a variety of other types of tumors in other types of medical imagery.

\section{4) In terms of cost of instrument}

We propose an automatic brain tumor detection and localization framework that can detect and localize brain tumor in magnetic resonance imaging. The proposed brain tumor detection and localization framework comprises five steps: image acquisition, pre-processing, edge. Detection, modified histogram clustering and morphological operations. After morphological operations, tumors appear as pure white color on pure black backgrounds. We used 50 neuro images to optimize our system and 100 out-of-sample neuro images to test our system. The proposed tumor detection and localization system was found to be able to accurately detect and localize Tempered image in magnetic resonance imaging. The preliminary results demonstrate how a simple machine learning classifier with a set of simple image-based features can result in high classification accuracy. 


\section{Advantages of SOM Over FCM and K- Means}

We have used self-organizing map (SOM) and a method of image processing to create a clustering mechanism that efficiently classifies image objects having an unknown probability distribution without requiring the determination of complicated parameters. This clustering mechanism is fast and highly reliable. The clustering method using the SOM promises to be a valuable tool for classifying large numbers of objects as it reduces the large data set by mapping it to a low dimensional map. It speeds up cluster processing. These algorithms are follow some step

Step 1: The input image in the form of JPEG is read after collection from the pathological lab.

Step 2: Conversion of the input RGB image into $\mathrm{L}^{*} \mathrm{a} \mathrm{b}^{*}$ * color space thereby storing the color information in the ' $\mathrm{a}$ *' and ' $\mathrm{b}$ *' layers.

Step 3: Application of the clustering techniques like $\mathrm{K}$ Means or the fuzzy $\mathrm{C}$ means is done on $\mathrm{a}^{*}$ and $\mathrm{b}^{*}$ layers which generates an output having $a^{*}$ and $b^{*}$ pixel values.

Step 4: Every pixel in the image is then labeled with the cluster index that which belongs to a corresponding cluster which is a result of K-Means or Fuzzy C-Means.

Step 5: The segmented images by color are created. The objects are separated in the image using the pixel labels

Step 6: ROI into separate image is segmented.

Step 7: Detected and marked with green color.

Step 8: The ROI boundary with the green mark is placed over the original image to highlight the ROI.

\section{Conclusion}

A novel approach has been proposed where a framework is designed that helps in identifying the ROI automatically which will be major breakthrough for medical practitioner. The framework uses fast and efficient clustering algorithm like K Means and Fuzzy C-Means which provide accurate results that can be made use to the optimum level by the human experts in order to determine any type of skin related diseases and share their knowledge regarding the same to the peer physiologists to counter it.

\section{References}

[1] "Automated ROI Detection for Histological Image using Fuzzy C-means and K-means algorithm” Rupesh Mandal, Mousumi Gupta, International Conference on Electrical, Electronics, and Optimization Techniques (ICEEOT) - 2016 Gunnar Lathen, "segmentation methods for medical image analysis,” Thesis No. 1434,

[2] Center for Medical Image Science and Visualization (CMIV)Linkoping University/US, SE-581 85 Linkoping, Sweden

[3] Dzung L. Pham, Chenyang Xu, Jerry L. Prince, “A survey of Current Methods in Medical Image Segmentation," Technical Report JHU/ECE 99-01; Annual Review of Biomedical Engineering(January 19,1998)

[4] Xiao-Yu Zhang, Jiu-Sheng Chen, Jian-Kang Dong, "Color Clustering Using Self- Organizing Maps,"
Proceedings of the 2007 International Conference on Wavelet Analysis and Pattern Recognition, Beijing, China, 2-4 Nov. 2007

[5] H.P. Ng, S.H. Ong, K.W.C. Foong, P.S. Goh, W.L. Nowinski, "Medical Image Segmentation Using KMeans Clustering And Improved Watershed Algorithm," 1-4244-0069-4/06/2006 IEEE.

[6] Keh-Shih Chuang, Hong-Long Tzeng, Sharon Chen, Jay Wu, Tzong-Jer Chen, "Fuzzy c- means clustering with spatial information for image segmentation," Computerized Medical Imaging and Graphics 30 (2006) 9-15.

[7] Quo Vadis, “Medical image segmentation”, computer methods and programs in biomedicine 84 ( 2006 ) 6365

[8] Nizar Grira, Michel Crucianu, Nozha Boujemaa, "Unsupervised and Semi-supervised Clustering: a Brief Survey,” INRIA Rocquencourt, B.P. 10578153 Le Chesnay Cedex, France. 